\title{
The Burden of Guardianship: A Matched Cohort Study
}

\author{
Daniel N. Ricotta, MD',2*, James J. Parris, MD, PhD'1, Ritika S. Parris, MD', \\ David N. Sontag, JD, M. Bioethics' ${ }^{1}$ Kenneth J. Mukamal, MD, MPH
}

${ }^{1}$ Beth Israel Deaconess Medical Center, Boston, Massachusetts; ${ }^{2}$ Carl J. Shapiro Institute for Education and Research, Boston, Massachusetts.

BACKGROUND: In cases where patients are unable to provide informed consent and have no surrogate decisionmaker, a hospital must seek guardian appointment as a legally recognized surrogate decision-maker.

OBJECTIVE: The aim of this study was to examine the magnitudes of length of stay (LOS) beyond medical clearance and healthcare costs among patients referred for guardianship.

DESIGN, SETTING AND PATIENTS: This was a retrospective cohort study of all 61 adult inpatients in a single tertiary care hospital requiring guardianship between October 1, 2014, and September 30, 2015, matched with up to three controls from the same discharging services and hospitalized for at least as long as the date of clearance for referred patients.

MEASUREMENTS: The following parameters were measured using generalized estimating equations: total LOS, LOS beyond medical clearance (excess LOS), medical complications, and total charges among referred patients, and the LOS and costs were compared with those of matched controls.

RESULTS: Mean LOS for patients requiring guardianship was $31 \pm 2$ days, and the total charges averaged $\$ 179,243 \pm$ 22,950 . We documented 12 hospital-acquired complications in 10 (16\%; $95 \%$ confidence interval [Cl], $8 \%-28 \%)$ unique patients. Accounting for potential confounders, the process of obtaining guardianship was associated with a $37 \%$ longer total LOS (95\% Cl [12\%-67\%]; $P=.002), 58 \%$ higher excess LOS (95\% Cl [2\%-145\%]; $P=.04)$, and $23 \%$ higher total charges (95\% Cl [4\%-46\%]; $P=.02$ ).

CONCLUSION: In this single-center cohort study, the guardianship process was associated with prolonged hospital stay and higher total hospital charges even when compared with matched controls. Furthermore, one in six patients suffered from a hospital-associated complication after medical clearance. Journal of Hospital Medicine 2018;13:595-601. Published online first February 5, 2018. (c) 2018 Society of Hospital Medicine

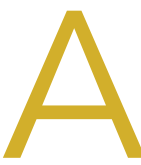

central tenet of modern medicine is that patients must provide fully informed consent to receive or refuse medical care offered by their clinical teams. ${ }^{1-4}$ If a patient is unable to make and communicate a choice or clearly indicate an understanding of the information presented, then he or she is considered to lack the capacity to make medical decisions and the medical team must seek consent from the patient's surrogate decision-maker. ${ }^{2-7}$ Every U.S. state recognizes a patient's healthcare proxy (HCP) and a court-appointed guardian as a legally recognized surrogate. 8,9 Most of the states also have statutes or regulations establishing a hierarchy of legally recognized surrogate decision-makers in the absence of a HCP or a court-appointed guardian, such as spouses, adult children, parents, siblings, and grandparents..$^{8,10}$

\footnotetext{
*Address for correspondence: Daniel Ricotta, MD, Carl J. Shapiro Institute for Education and Research, 330 Brookline Ave, ES-2, Boston, MA 02215; Telephone: 617-667-9120; Fax: 617-667-9122; E-mail:dricotta@ bidmc.harvard.edu

Additional Supporting information may be found in the online version of this article.
}

Received: September 15, 2017; Revised: December 11, 2017; Accepted: December 17, 2017

() 2018 Society of Hospital Medicine DOI 10.12788/jhm.2946
In states that do not have such a statute, hospitals develop their own institutional policies for surrogate decision-making.

However, there are important limitations on the authority of these surrogate decision-makers. ${ }^{10}$ For instance, patients may not have a family member or a friend to serve as a surrogate decision-maker, often family members cannot override a patient's objection, even when that patient lacks decision-making capacity, and certain decisions require a guardian or a HCP.8${ }^{10}$ In these circumstances, the hospital must petition a court to appoint a guardian as a legally recognized surrogate decision-maker. This can be an involved family member, if one exists, or an independent, typically volunteer, guardian. ${ }^{11}$ The process of guardian appointment is complex $x^{7,11}$ and can range from a few days to more than a month, largely dependent on court dates and finding a volunteer guardian. Much of the process occurs during the patient's hospital stay. This prolongation of hospitalization would be expected to increase health care costs and iatrogenic complications, ${ }^{12-14}$ but data quantifying these for patients requiring guardianship are lacking.

The goal of this study was to describe the characteristics of patients who undergo the process of guardianship and measure the associated burdens. These burdens include the financial costs to the medical system, the prolonged length of stay beyond medical necessity, and the costs to the patient in the form of hospital-acquired complications. Investigating the bur- 
den of guardianship is an important first step in uncovering opportunities to improve the process. We hypothesized that patients requiring guardianship would have lengths of stay and healthcare costs that were at least as large as those for patients whose conditions required similar durations of hospitalization prior to medical clearance, in part due to iatrogenic complications that would accrue while awaiting guardian appointment.

\section{METHODS}

\section{Setting}

We conducted a retrospective matched cohort study of adult inpatients at Beth Israel Deaconess Medical Center (BIDMC), a 651-bed academic, tertiary care facility in Boston, Massachusetts. The study was approved by the BIDMC Institutional Review Board as a nonhuman subject research consistent with hospital operations.

\section{Population}

For this matched cohort study, we identified case patients as those hospitalized for any reason for whom guardianship proceedings were initiated and obtained; only the first hospitalization during which the guardianship was pursued was used. Cases were identified by obtaining the data of all patients for whom the BIDMC general counsel completed the process of guardianship between October 2014 and September 2015. At BIDMC, all the guardianship proceedings are referred to the general counsel.

To determine the postclearance experience for referred patients compared with that for other patients with similar lengths of stay up to those of the referred patients' point of clearance, we identified up to three matched controls for each case (Supplemental Figure 1). Medical clearance was defined as the date when the patient was medically stable to be discharged from the hospital, and it was determined in an iterative manner. We identified controls as hospitalized patients admitted for any cause and matched to the cases requiring guardianship on discharging service and length of stay prior to clearance. Specifically, we identified patients on the same service as the case whose length of stay was at least as long as the length of stay of the case patient until medical clearance, as defined below. We then determined the total and the excess length of stay, defined as the duration beyond clearance for each case referred for guardianship; for controls, the 'excess' length of stay was the number of hospitalized days beyond the corresponding time that a matched case had been provided clearance. To account for seasonal influences and the training level of house officers, we selected the three controls whose discharge date was closest (before or after) to the discharge date of their matched case.

From legal team files, we identified 61 patients hospitalized at BIDMC for whom new guardianship was pursued to completion. Of these 61 patients, 10 could not be matched to an appropriate control and were included in descriptive analyses but not in comparisons with controls.

\section{Covariates and Outcomes}

We collected the details regarding age, gender, primary language, highest level of education, marital status, insurance status, race, date of admission, date of discharge, discharge disposition, principal diagnosis, case mix index (CMI), and discharging service from our administrative and billing data. Outcomes of interest included length of stay and total hospital charges that were collected from the same databases. We used hospital charges, rather than payments, to ensure uniformity across payers.

\section{Chart Review}

Unique to cases, a team of two medical residents (J.P., R.P.) and a hospitalist (D.R.) determined the date of medical clearance and hospital-associated complications by a chart review. The date of medical clearance was then used to calculate excess length of stay, ie, the duration of stay beyond the date of medical clearance, by subtracting the time to medical clearance from the total inpatient length of stay.

We developed a novel algorithm to determine the date of medical clearance consistently (Figure 1). We first determined whether the discharge summary indicated a clear date of medical readiness for discharge. If the discharge summary was unclear, then a case management or a social work note was used. The date of medical clearance determined by the case management or the social work note was then confirmed with clinical data. The date was confirmed if there were no significant laboratory orders and major medication changes or procedures for 24 hours from the date identified. If notes were also inconclusive, then the medical clearance was determined by a review of provider order entry. Medical readiness for discharge was then defined as the first day when there were no laboratory orders for 48 hours and no significant medication changes, imaging studies, or microbiologic orders.

Hospital-acquired complications were determined to be related to the guardianship process if they occurred after the date of medical stability but prior to discharge. We did not investigate hospital-acquired complications among controls. Hospital-acquired complications were defined as follows:

- Catheter-associated urinary tract infection (CAUTI): active Foley catheter order and positive urine culture that resulted in antibiotic administration.

- Hospital-acquired pneumonia (HAP): chest X-ray or computed tomography (CT) scan showing a consolidation that resulted in antibiotic administration.

- Venous thromboembolism (VTE): positive venous ultrasound or CT angiography of the chest for deep venous thrombosis (DVT) or pulmonary embolism (PE).

- Decubitus ulcer: new wound care consultation for sacral decubitus ulceration.

- Clostridium difficile (C. diff) infection: positive stool polymerase chain reaction that resulted in antibiotic administration.

The algorithm for identifying the date of clearance and the presence of complications was piloted independently by three investigators (R.P., J.P., D.R.) using a single chart review and was redesigned until a consensus was obtained. The same three investigators then independently reviewed three additional charts, including all notes, laboratory results, imaging results, and orders, with complete agreement for both date 


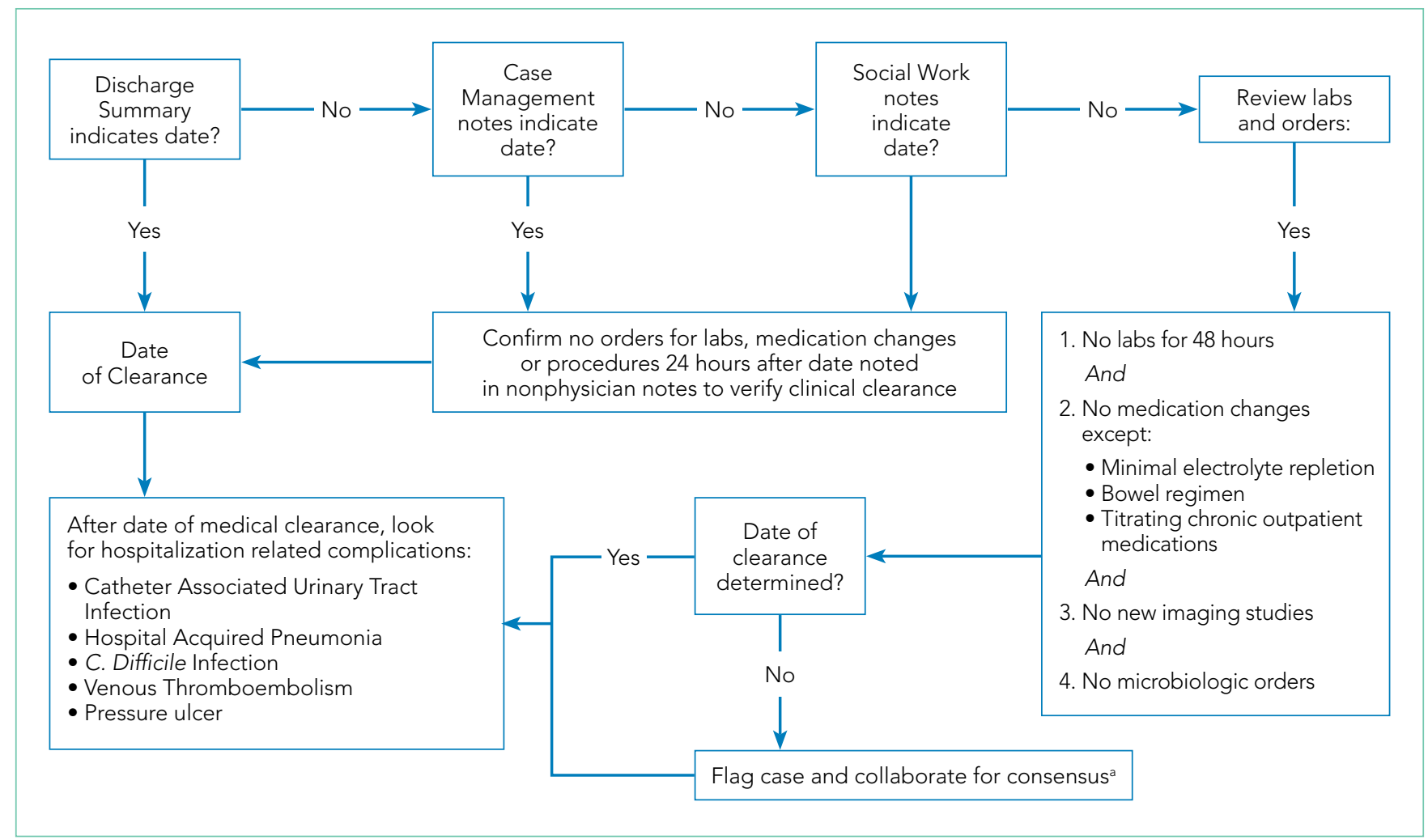

FIG 1. Algorithm for determining medical clearance. Algorithm used to determine date of medical clearance and presence of complications among patients referred for guardianship.

aNo cases were flagged

of clearance and presence of complications. Two investigators (R.P., J.P.) then individually reviewed the remaining 57 charts. Of these, 10 were selected a priori for review by both investigators for interrater reliability, with a mean difference of 0.5 days in the estimated time to clearance and complete concordance in complications. In addition, a third investigator (DR) independently reread 5 of the 57 reviewed charts, with complete concordance in both time to clearance and presence of complications with the original readings.

\section{Statistical Analysis}

SAS 9.3 was used for all analyses (SAS Institute Inc., Cary, North Carolina).

We first examined the demographic and clinical characteristics of all 61 patients who underwent guardianship proceedings. Second, we described the primary outcomes of interestlength of stay, costs, and likelihood of complications - in this series of patients with associated $95 \%$ confidence intervals.

Third, we examined the associations between guardianship and length of stay and healthcare costs using generalized estimating equations with clustering by matched set and compound symmetry. For length of stay, we specifically assessed excess length of stay (the matching variable) to avoid immortal time bias; we also examined the total length of stay. For all regression analyses, we adjusted for the following covariates: age, gender, education, marital status, race/ethnicity, CMI, insurance status, discharging service, and principal diagnosis. To maximize normality of residuals, costs were log-transformed; length of stay beyond clearance was log-transformed after addition of one. For both outcomes, we back-transformed the regression coefficients and presented percent change between case and control patients. All reported tests are two-sided.

\section{RESULTS}

A total of 61 guardianship cases and 118 controls were included in the analysis.

\section{General Characteristics}

The characteristics of all cases prior to matching are included in Table 1. The department of internal medicine discharged the largest proportion of cases, followed by neurosurgery and neurology departments. More than $65 \%$ of cases were insured by Medicare or Medicaid. Three-quarters of cases were discharged from the hospital to another medical facility, with about half discharged to a skilled nursing facility (SNF) or a rehabilitation center and one-quarter to a long-term acute care hospital (LTACH).

The median length of stay for patients requiring guardianship was 28 (range, 23-36) days, and the median total charges were $\$ 171,083(\$ 106,897-\$ 245,281)$, with a total cost approximating $\$ 10.9$ million for these patients. Regarding hospital-acquired complications, 10 (16\%; 95\% confidence interval, $8 \%-28 \%$ ) unique cases suffered from a complication, with HAP being the most frequently $(n=5)$ occurring complication. 
TABLE 1. Characteristics of Patients Referred for Guardianship

\begin{tabular}{lc}
\hline Variable & $\mathbf{N}=\mathbf{6 1}$ \\
\hline Age (years) & $61(52-72)$ \\
\hline Case Mix Index & $5.4(1.7-10.9)$ \\
\hline Total ICU Stay (days) & $13(2-22)$ \\
\hline \# Discharges in Last Year & $1(1-3)$ \\
\hline Total Length of Stay (days) & $28(23-36)$ \\
\hline Total Charges (\$) & $\$ 171,083(\$ 106,897-\$ 245,281)$ \\
\hline Discharging Service & \\
Surgery & $5(8 \%)$ \\
Internal medicine & $25(41 \%)$ \\
Neurosurgery & $19(31 \%)$ \\
Neurology & $11(18 \%)$ \\
Psychiatry & $1(2 \%)$ \\
\hline Gender & \\
F & $35(57 \%)$ \\
\hline Complications & \\
CAUTI & $3(5 \%)$ \\
DVT & $1(2 \%)$ \\
Fall & $1(2 \%)$ \\
HAP & $5(8 \%)$ \\
Sacral Ulcer & $2(3 \%)$ \\
None & $51(84 \%)$ \\
\hline & \\
\hline & \\
\hline
\end{tabular}

\begin{tabular}{lc}
\hline Ethnicity & \\
White & $31(51 \%)$ \\
Black & $4(7 \%)$ \\
Hispanic & $3(5 \%)$ \\
Asian & $3(5 \%)$ \\
Other/Multiracial/Unknown & $20(33 \%)$
\end{tabular}

\begin{tabular}{ll}
\hline Education & \\
College & $14(23 \%)$ \\
High School & $24(39 \%)$ \\
Unknown & $23(38 \%)$ \\
\hline
\end{tabular}

\begin{tabular}{lc}
\hline Primary Language \\
English \\
Non-English & $56(92 \%)$ \\
\hline Marital Status & $5(8 \%)$ \\
Married & \\
Divorced & $12(20 \%)$ \\
Single & $6(10 \%)$ \\
Widowed & $22(36 \%)$ \\
Unknown & $4(7 \%)$ \\
\hline Disposition & $17(28 \%)$ \\
Home & \\
Rehab/SNF & $2(3 \%)$ \\
LTACH & $32(52 \%)$ \\
Inpatient Psychiatry & $16(26 \%)$ \\
Expired & $5(8 \%)$ \\
\hline
\end{tabular}

Insurance Status

$\begin{array}{lc}\text { Private } & 16(26 \%) \\ \text { Medicare } & 27(44 \%) \\ \text { Medicaid } & 13(21 \%) \\ \text { Other } & 5(8 \%)\end{array}$

*Data are represented as median (IQR) for continuous variables and $\mathrm{N}(\%)$ for nominal variables

Abbreviations: CAUTI, catheter-associated urinary tract infection; DVT, deep venous thrombosis; HAP, hospital-acquired pneumonia; ICU, intensive care unit; LTACH, longterm acute care hospital; SNF: skilled nursing facility.

\section{Comparison with Matched Controls}

No statistically significant differences were observed between cases and controls in terms of age, primary language, highest level of education, ethnicity, insurance status, or discharging service as shown in Table 2; discharging service was a matched variable and comparable by design. However, cases tended to be less likely to be married and had a higher CMI.

When compared with control patients in terms of similar services who stayed for at least as long as their duration to clearance, the cases had significantly longer lengths of stay compared to those of controls (29 total days compared to 18 days, $P<.001$; Figure 2). In addition, cases incurred significantly higher median total charges $(\$ 168,666)$ compared to those of controls $(\$ 104,190 ; P=.02)$.

After accounting for potential confounders, including age, gender, language, education, marital status, discharging service, ethnicity, insurance status, CMI, and principal diagnosis, guardianship was associated with 58\% higher excess length of stay $(P=.04,95 \% \mathrm{Cl}[2 \%-145 \%])$. Furthermore, guardianship was associated with $23 \%$ higher total charges $(P=.02,95 \% \mathrm{Cl}$ $[4 \%-46 \%])$ and $37 \%$ longer total length of stay $(P=.002,95 \%$ Cl [12\%-67\%]).

\section{DISCUSSION}

In this cohort study of 61 inpatients from a single academic medical center who needed guardianship, patients who required this process had prolonged lengths of stay and substantial healthcare costs even when compared with matched controls who stayed at least as long as the cases' date of clearance. One in six patients suffered from hospital-associated complications after their date of medical clearance.

To our knowledge, this is among the first studies to investigate healthcare costs and harm to the patient in the form of hospital-associated complications as a result of guardianship proceedings. Other studies ${ }^{15,16}$ have also demonstrated excessive length of stay attributed to nonclinical factors such as guardianship, though they did not quantify the excess stay or compare guardianship cases with a matched control. One study ${ }^{17}$ demonstrated total charges of $\$ 150,000$ per patient requiring guardianship, which are similar to our results. However, Chen et al. also observed an average of 27.8 medically unnecessary days, which are 16 more days than those in our study sample. This may reflect the difference in how excess days were determined, namely, statistical process control analysis in the previous study compared with a manual chart review in our study. To our knowledge, no other study has compared guardianship cases with matched controls to compare their experiences to patients with similarly prolonged stays prior to clearance.

After matching by service and the length of stay until medical clearance in each guardianship case, the subsequent length of stay was higher among cases than among controls, even after adjustment for differences in $\mathrm{CMl}$ and diagnosis. This suggests that the process of obtaining guardianship results in a particularly prolonged length of stay, which is presumably attributable to factors other than medical complexity or ongoing illness. 
TABLE 2. Characteristics of Patients Referred for Guardianship and Matched Controls ${ }^{a}$

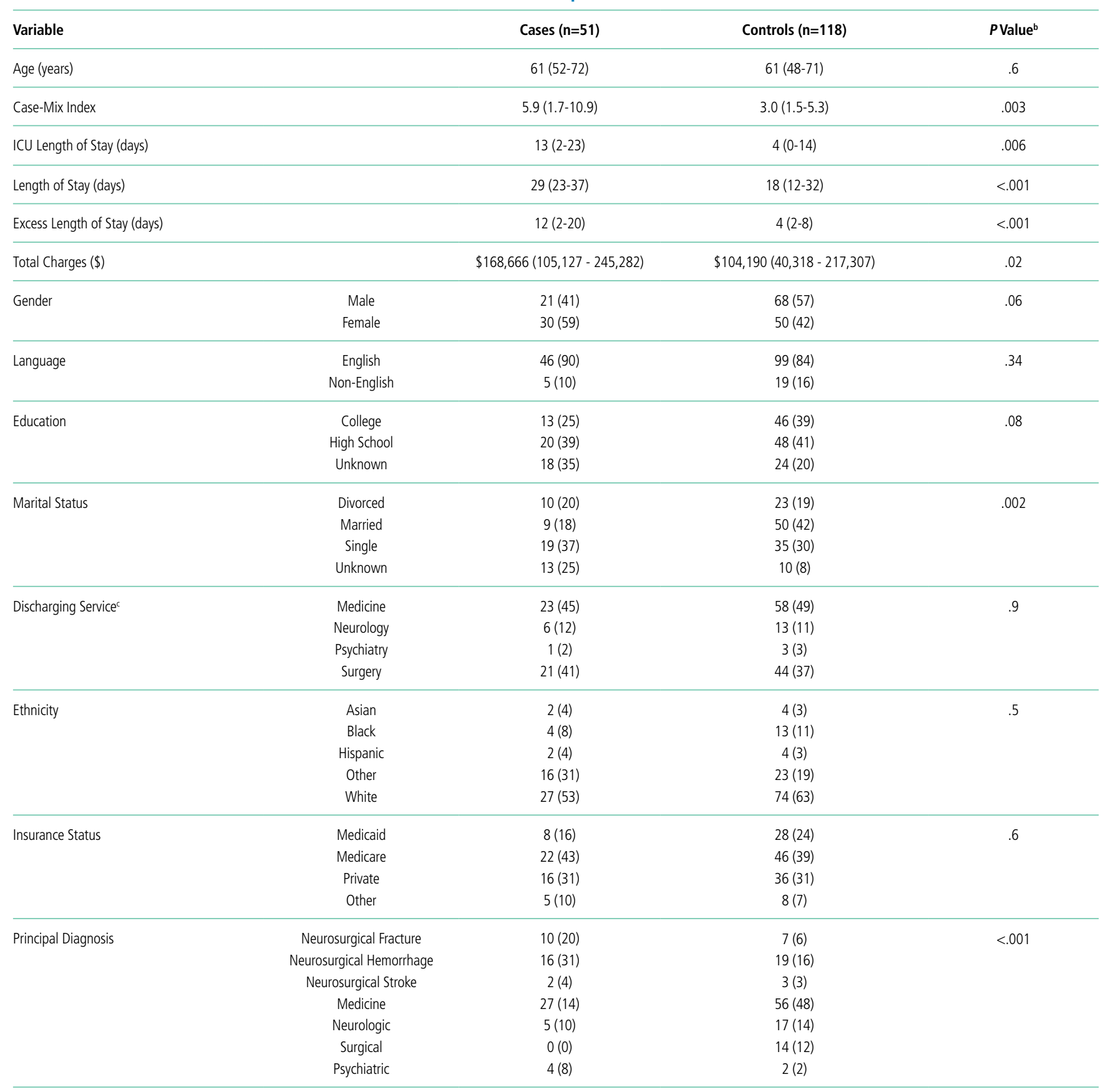

aData are represented as median (IQR) for continuous variables and $\mathrm{N}(\%)$ for nominal variables

${ }^{b} P$ values calculated using Wilcoxon two-sample t-test for continuous variables and Fisher's Exact for nominal variables

cReferred cases and controls matched on discharge service and length of stay prior to clearance.

It is probable that at least two interrelated mechanisms are responsible for the particularly high costs and the long stay of patients who require guardianship. First, the process of obtaining guardianship is itself protracted in several cases, necessitating long-term admissions well beyond the point of medical stability. Second, our results suggest that longer hospital stays are apt to grow further in a feed-forward cycle due to hospital-acquired complications that develop after the date of med- ical clearance. Indeed, in our series, $16 \%$ of patients sustained a complication that is readily attributable to hospital care after their date of clearance, and these types of complications are likely to lengthen the stay even further.

We compared cases referred for guardianship to control patients on the same services, at similar time points, whose length of stay was at least as long as the point of medical clearance as their corresponding case patient. Because cases were hospi- 


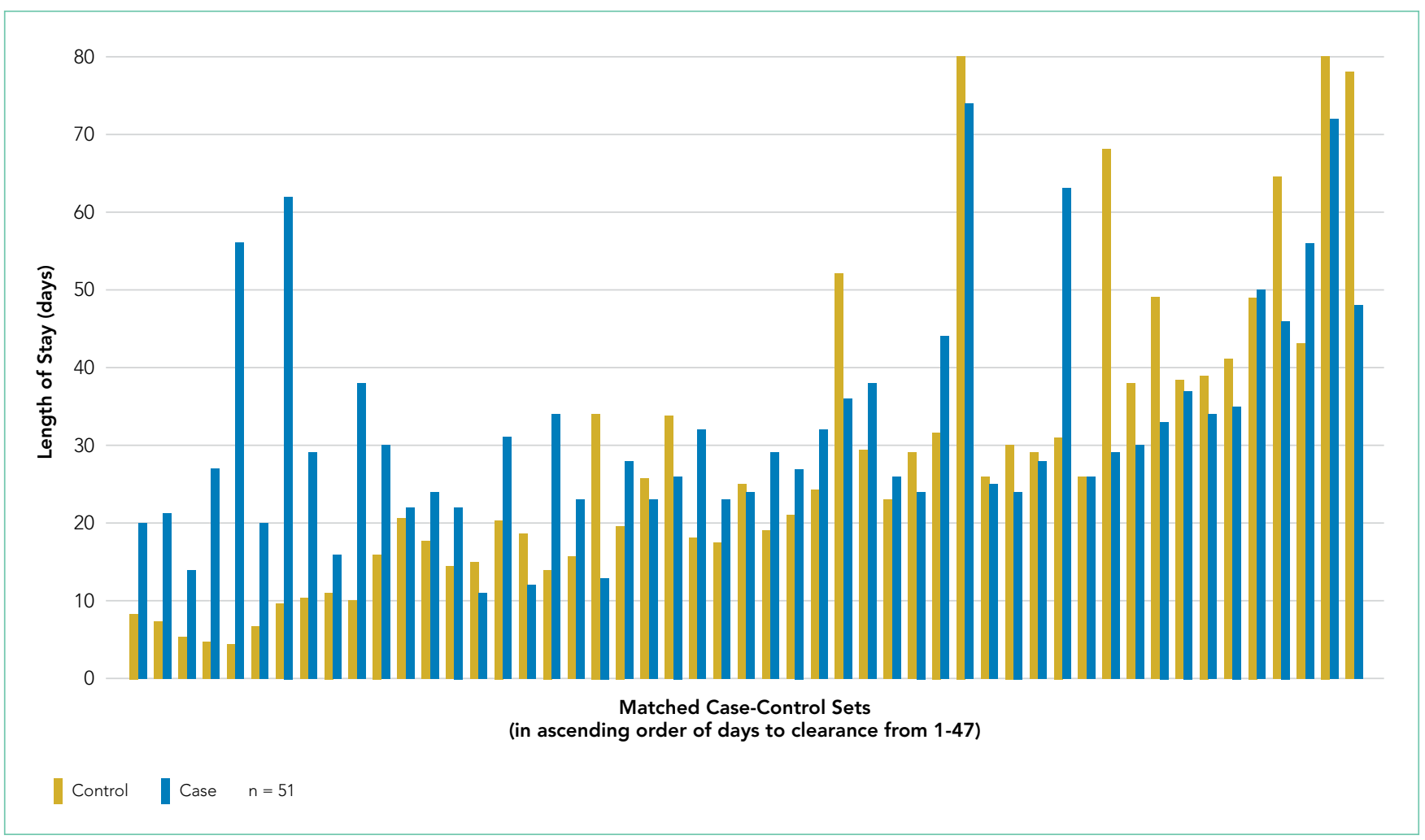

FIG 2. Total length of stay among patients referred for guardianship ( $n=51)$ and matched controls. Sets presented in ascending order of time to clearance among cases. Blue bars indicate case patients referred for guardianship, and gold bars indicate the mean among up to 3 matched controls.

talized with active medical needs to at least the point of clearance, we anticipated that costs might well be lower among cases, who had no medical necessity for hospitalization at the point of clearance, compared with controls who remained hospitalized presumably for active medical needs. Counter to this hypothesis, and accounting for potentially confounding variables, undergoing a guardianship proceeding was associated with nearly $25 \%$ higher costs of patient care. This may ultimately represent a substantial burden on the healthcare system. For example, in just one year in our hospital, the total hospital charges reached almost \$11 million for the 61 patients who underwent guardianship proceedings. Considering that $65 \%$ of the patients requiring guardianship had Medicaid or Medicare coverage, there are significant financial implications for the hospital systems and to the public.

Limitations of our study relate to its retrospective nature at a single center. Investigating guardianship cases at a single center and with a small sample size of 61 patients limits generalizability. Nevertheless, we still had enough power to detect significant differences compared with matched controls, and, to our knowledge, this study remains the largest investigation into the cost associated with guardianship to date and the only study comparing guardianship cases with matched controls. Furthermore, we did not complete chart reviews of controls, which limits direct comparisons of complications and precluded our matching on variables that required detailed review.

The retrospective design may include confounders unaccounted for in our statistical design, though we attempted to match cases with controls to account for some of these potential differences and included a broad set of covariates that included measures of comorbidity and diagnosis. To this point, we included only $\mathrm{CMI}$ and principal diagnosis as the measures of severity, and adjustment for $\mathrm{CMI}$, which includes features of the index hospitalization itself, may represent overadjustment. However, this type of overadjustment would tend to bias toward the null hypothesis.

Investigators only completed chart reviews for cases, which limits our ability to contrast the rate of hospital-associated complications for cases with that of controls. However, the rates of CAUTI and HAP complications among our cases were notably higher than national inpatient estimates, ie, $5 \%$ and $8 \%$ compared to $0.2 \%{ }^{18}$ and $0.5 \%-1 \%,{ }^{19}$ respectively. Furthermore, we demonstrated higher total costs and total lengths of stay among guardianship patients, analyses for which the attributed date of clearance for controls was not required, and the rate of complications among the case patients was sizable despite their being formally medically cleared. In other words, regardless of whether a complication rate of $16 \%$ is "typical" for inpatients hospitalized for these durations, this suggests that persistent hospitalization after clearance does not carry a benign prognosis.

In addition, to estimate healthcare costs, we relied on total hospital charges, which are readily available and reflect, at least in part, payer costs but do not reflect true costs to the medical center. Nonetheless, charges approximately reflect costs - with some variation across cost centers - and hence 
provide a useful metric for comparing cases and controls. To provide context, for academic medical centers such as ours, costs are typically about half of charges.

Finally, each state has different statutes for surrogate decision making. The results of this study reflect the Massachusetts' experience, with no public guardianship program or hierarchy statute. That being said, while this presumably causes the need for more guardianships in Massachusetts, the mechanisms for guardianship are broadly similar nationwide and are likely to result in excessive length of stay and cost similar to those in our population, as demonstrated in studies from other states. ${ }^{7,15-17}$

\section{Implications}

At a time where medical systems are searching for opportunities to reduce the length of stay, prevent unnecessary hospitalization, and improve the quality of care, reevaluating the guardianship process is ripe with opportunity. In this single academic center, the process of guardianship was associated with $58 \%$ excess length of stay and $23 \%$ higher total hospital charges. Furthermore, one in six patients requiring guardianship suffered from hospital-associated complications.

\section{References}

1. O'Neill O. Autonomy and Trust in Bioethics. Cambridge: Cambridge University Press; 2002.

2. Beauchamp T, Childress J. Principles of Biomedical Ethics. 7th ed. New York: Oxford University Press; 2013.

3. McMurray RJ, Clarke OW, Barrasso JA, et al. Decisions near the end of life. J Am Med Assoc. 1992;267(16):2229-2233.

4. American Medical Association. AMA Principles of Medical Ethics: Chapter 2 - Opinions on Consent, Communication and Decision Making.; 2016.

5. Arnold RM, Kellum J. Moral justifications for surrogate decision making in the intensive care unit: Implications and limitations. Crit Care Med. 2003;31(Supplement):S347-S353.

6. Karp N, Wood E. Incapacitated and Alone: Healthcare Decision Making for Unbefriended Older People. Am Bar Assoc Hum Rights. 2003;31(2).

7. Bandy RJ, Helft PR, Bandy RW, Torke AM. Medical decision-making during the guardianship process for incapacitated, hospitalized adults: a descriptive cohort study. J Gen Intern Med. 2010;25(10):1003-1008.

8. Wynn S. Decisions by surrogates: an overview of surrogate consent laws in the United States. Bifocal. 2014;36(1):10-14.

9. Massachusetts General Laws. Chapter 201D: Health Care Proxies. https:// malegislature.gov/Laws/GeneralLaws/Partll/Titlell/Chapter201D. Published 2017. Accessed March 31, 2017.

10. American Bar Association Commision on Law and Aging. Default Surrogate Consent Statutes. Am Bar Assoc. 2016:1-17.

11. Massachusetts General Laws. Chapter 190B: Massachusetts Probate Code.
This matched cohort study adds quantitative data demonstrating substantial burdens to the healthcare system as a result of the guardianship process and can be used as an impetus for hospital administration and legal systems to expedite the process. Potential improvements include increasing HCP form completions (which would eliminate the need to pursue guardianship for most of such patients), identifying patients who lack a legally recognized surrogate decision-maker earlier in their hospital stay (ideally upon admission), and providing resources to assist clinical teams in the completion of affidavits necessary to support the appointment of a guardian, so that paperwork can be filed with courts sooner. Further research that provides more generalizable prospective data could potentially improve the guardianship process and reduce its burden on hospitals and patients even further.

\section{Acknowledgments}

The authors express their tremendous thanks to Gail Piatkowski for her invaluable assistance in collecting administrative and billing data.

Disclosures: The authors report no conflicts of interest. The authors alone are responsible for the content and writing of the article

https://malegislature.gov/Laws/GeneralLaws/Partll/Titlell/Chapter190B. Published 2017. Accessed March 31, 2017.

12. Rosman M, Rachminov O, Segal O, Segal G. Prolonged patients' in-hospital waiting period after discharge eligibility is associated with increased risk of infection, morbidity and mortality: a retrospective cohort analysis. BMC Health Serv Res. 2015;15:246.

13. Majeed MU, Williams DT, Pollock R, et al. Delay in discharge and its impact on unnecessary hospital bed occupancy. 2012.

14. Nobili A, Licata G, Salerno F, et al. Polypharmacy, length of hospital stay, and in-hospital mortality among elderly patients in internal medicine wards. The REPOSI study. Eur J Clin Pharmacol. 2011;67(5):507-519.

15. Chen JJ, Finn CT, Homa K, St Onge KP, Caller TA. Discharge delays for patients requiring in-hospital guardianship: A Cohort Analysis. J Healthc Qual. 2016;38(4):235-242.

16. Chen JJ, Kwon A, Stevens Y, Finn CT. Barriers beyond clinical control affecting timely hospital discharge for a patient requiring guardianship. Psychosomatics. 2015;56(2):206-209.

17. Chen JJ, Blanchard MA, Finn CT, et al. A clinical pathway for guardianship at dartmouth-hitchcock medical center. Jt Comm J Qual Patient Saf. 2014;40(9):389-397.

18. McEachern R, Campbell Jr GD. Hospital-Acquired Pneumonia: Epidemiology, Etiology, and Treatment. Infect Dis Clin North Am. 1998;12(3):761-779.

19. Zimlichman E, Henderson D, Tamir O, et al. Health care-associated infections. JAMA Intern Med. 2013;173(22):2039. 